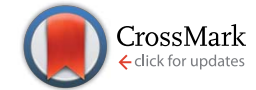

Cite this: RSC Adv., 2017, 7, 8118

Received 15th November 2016

Accepted 6th January 2017

DOI: $10.1039 / c 6 r a 26849 h$

www.rsc.org/advances

\section{Stability, rheological property and oil-displacement mechanism of a dispersed low-elastic microsphere system for enhanced oil recovery}

\author{
Hongbin Yang, ${ }^{\text {ab }}$ Wanli Kang, ${ }^{\text {*ab }}$ Hairong Wu, ${ }^{a}$ Yang Yu, ${ }^{a}$ Zhou Zhu, \\ Pengxiang Wang, ${ }^{\mathrm{b}}$ Xiangfeng Zhang ${ }^{\mathrm{b}}$ and Bauyrzhan Sarsenbekuly ${ }^{\mathrm{b}}$
}

\begin{abstract}
A dispersed low-elastic microsphere system, consisting of low-viscoelastic microspheres and polymers, is a novel suspension system for enhanced oil recovery in heterogeneous reservoirs. In this study, experiments were performed to characterize the morphology, viscoelasticity, and swelling performance of the synthetic low-elastic microspheres. The stability and rheological property of the dispersed low-elastic microsphere system was investigated using a Turbiscan Lab Expert stability analyzer and an MCR301 rheometer. In the flow and displacement experiments, the parallel-sandpacks model and microscopic visualization models were used to study the oil-displacement effect and micromigration mechanism of the low-elastic microspheres. The experimental results showed that the storage modulus $\left(G^{\prime}\right)$ of the synthetic lowelastic microspheres was only $23.6 \mathrm{~Pa}$ and they also had a good swelling property in the simulated formation water. The parallel-sandpacks test and micromodel test indicated that the dispersed lowelastic microsphere system was a promising agent for both conformance control and improved oil recovery in heterogeneous reservoirs. Moreover, the low-elastic microspheres had good deformation and shear resistance performances. Five transport behaviors, such as deformable passing through, partition passing through, blockage, adhesion, and directly passing through, of low-elastic microspheres in a porous medium have been put forward. This study not only provides an understanding of the properties of the dispersed low-elastic microsphere system but also supplies theoretical support for the mechanism of improving oil recovery for the dispersed low-elastic microsphere system.
\end{abstract}

\section{Introduction}

With the development of mature oilfields, the phenomenon of water breakthrough among oil wells and water wells is becoming more and more serious. And, the injection of water for oil recovery is not effective. Thus, there is a large amount of oil remaining in the reservoirs. As the number of newly discovered reservoirs decreases, it is more and more important to improve the oil recovery of mature oilfields. According to the previous reports, reservoir heterogeneity is one of the most important factors for low-oil recovery and water breakthrough. ${ }^{\mathbf{1 , 2}}$ Reservoirs with induced fractures or high permeability channels, commonly called thief zones or streaks due to extensive water flooding, are quite common in mature reservoirs. Many methods, including polymer flooding, alkalinesurfactant-polymer flooding, foam flooding, and gel

${ }^{a}$ Enhanced Oil Recovery Research Institute, China University of Petroleum (Beijing), Beijing 102249, People's Republic of China. E-mail: hongbinyang@upc.edu.cn; kangwanli@126.com

${ }^{b}$ School of Petroleum Engineering, China University of Petroleum (East China), Qingdao 266580, People's Republic of China treatments, have been applied to mitigate water breakthrough and to improve oil recovery.

Many polyacrylamide polymer gels have been used to reduce the water cut by sealing the thief zone and redistributing the water drive to enhance the oil recovery of heterogeneous oilfields. ${ }^{3-8}$ Although in situ polyacrylamide polymer gels are economically attractive, they have some inherent negative effects on the treatment of wells caused by the formation temperature, salinity, shearing, adsorption, diluted formation water, chromatographic separation effect or other environmental factors. Considering the abovementioned negative effects, preformed gels have been proposed for conformance control and improving oil recovery. The preformed gels are formed at surface facilities before injection, whereby they are injected into the reservoir in the form of particles. These particle gels can tolerate high salinity and high temperature, but are slightly sensitive to $\mathrm{pH}$ and $\mathrm{H}_{2} \mathrm{~S}$ reservoir conditions. ${ }^{9-11}$ Preformed gels can be divided into microgels, preformed particle gel (PPG), and Bright Water according to their particle size, swelling ratio, and swelling time. In 2001, Chauveteau et al. prepared a type of microgel using a terpolymer of acrylamide containing $2 \%$ acrylates and $2 \%$ sulfonated groups as a monomer and zirconium(Iv) lactate as a cross-linker. ${ }^{12}$ The diameter 
of typical microgels was 1-3 $\mu \mathrm{m}$. Zaitoun et al. researched the migration of microgels in a porous medium and found that there was no sign of continuous plugging in high permeability or fractural reservoirs. ${ }^{13}$ Furthermore, both Choi et al. and Benson et al. reported that a high filtration retention of polyelectrolyte microgels occurred in mid-permeability rocks. This problem was solved by the pre-addition of citric acid. However, low-pH conditions could increase the polymer adsorption near the wellbore and impose additional chemical costs. ${ }^{\mathbf{1 4 , 1 5}}$ Bright Water was proposed by both Pritchett et al. and Frampton et al. The diameter of this gel system varied from dozens of microns to a few hundred microns. The gel system was prepared by the reversed phase emulsification polymerization of acrylamide and its derivatives with both labile and stable cross-linkers. Unfortunately, due to poor thermal stability and a limited application range, Bright Water was not widely used in a pilot. ${ }^{16,17}$ PPG was initiated by Petro China in 1996, and was prepared by a solution polymerization method, followed by crushing and sieving to the desired particle size using acrylamide and $N, N^{\prime}$-methylenebisacrylamide as the monomer and cross-linker, respectively. The particle size of PPG ranges from millimeters to micrometers. Millimeter-size PPG has been successfully used in more than 5000 wells in China for deep profile control performance. ${ }^{18}$ PPGs were also employed by Halliburton and Occidental to control $\mathrm{CO}_{2}$ breakthrough in $\mathrm{CO}_{2}$ flooding areas. ${ }^{19}$ PPG can tolerate high salinity and high temperature and is thus can support the development of oilfields with strong heterogeneity, a high water-cut, and large porous channels. ${ }^{20,21}$ However, the application of PPG is limited to reservoirs with fractures or fracture-like channels because of its large size. ${ }^{22}$ In addition, PPGs have no defined shape and are easily broken after swelling in fractures.

In the early 21st century, a new chemical agent called a viscoelastic microsphere was proposed and has attracted extensive interest from researchers. ${ }^{23-25}$ Some pilot tests were performed in Shengli, Jidong, and Dagang Oilfields in China and a good effect of water control and enhanced oil recovery was obtained. ${ }^{26-29}$ The viscoelastic microsphere used in petroleum engineering was prepared by inverse phase polymerization, with a particle size ranging from several nanometers to hundreds of micrometers based on the pore characteristics of the target layer in underground conditions. Viscoelastic microspheres are a viscoelastic plugging agent with a $3 \mathrm{D}$ structure, and can absorb much more water as compared to their own mass, ranging from several to hundreds of times more, and furthermore, it is hard to release the absorbed liquids even under high pressure. ${ }^{30,31}$ However, despite the many advantages it has, there are still some problems in the practical application in oilfields. The high rigidity of industrial viscoelastic microspheres leads to a poor deformability. Consequently, industrial viscoelastic microspheres are retained around the near-wellbore area. After absorption and swelling, they might be sheared and broken, limiting the blocking effect. Hence, a kind of viscoelastic microsphere that can be flexible during injection and resistive to shearing after swelling is in urgent need to meet the requirements of injection and blocking.
Although the deformability is one of the most important properties for successful water control and enhanced oil recovery, few studies have investigated the effect of the storage modulus $\left(G^{\prime}\right)$ and loss modulus $\left(G^{\prime \prime}\right)$ of viscoelastic microspheres on the profile control and oil-displacement behavior. Moreover, the stability and rheological properties of the dispersed low-elastic microsphere system have not been comprehensively researched before. The stability of the dispersed low-elastic microsphere system plays a decisive role in deep profile control. If the stability is poor, the purpose of deep profile control would not be achieved easily as viscoelastic microspheres can easily adsorb in near the wellbore and plug the injection channel near the wellbore. The assessment method for stability of the dispersion system is a direct observation of the particle sedimentation of the dispersion system by the naked eye; for example, the bottle test method. ${ }^{32}$ This method is simple and popular, but the results of this method are qualitative and there are certain subjective factors that need to be taken into consideration. Currently, optical analysis instruments have been used to analyze the stability of the suspension system. The TURBISAN Lab Expert type stability analyzer is one kind of such instrument, which uses multiple light scattering techniques to study the stability. It can analyze microscopic phenomena of emulsions, suspensions, foam liquids, and other dispersion systems quickly. Besides, it can analyze the rate of sedimentation or flotage and changes in internal phase particle size quantitatively. It has been widely used to analyze the stability of coal-water slurry, pesticides, food, crude oil emulsions, and others. ${ }^{33-36}$ Surprisingly, the stability of the dispersed swellable viscoelastic microspheres system has not been reported yet. Polymer microspheres are different from conventional rigid particles in that the morphology of polymer microspheres can change with the imposition of a weak external force. The dispersed polymer microsphere system is known as a complex fluid. The rheology of the dispersed system can reflect the change in internal structure of the fluid. The rheological measurement of the dispersed system has been an effective method to detect their complex structure. Research into the rheology of the dispersed system has mainly focused on issues around agricultural chemicals, papermaking, and the food industry. The rheology of the dispersed polymer microspheres is particularly complicated because of the charge on the surface of the microspheres and the swelling property. ${ }^{37,38}$ Currently, very few reports about the rheological properties of the dispersed polymer microspheres system have been taken.

In the present work, a low-elastic microsphere with a storage modulus $\left(G^{\prime}\right)$ of 23.6 Pa was prepared through inverse suspension polymerization. The macroscopic features and internal structure were characterized, then the swelling and viscoelasticity of microspheres in simulated formation water were studied. A Turbiscan Lab Expert type stability analyzer and MCR301 rheometer were used to investigate the stability and rheological property of the dispersed low-elastic microsphere system. The micro-migration mechanism and oil-displacement mechanism were then studied using one micromodel and a series of parallel-sandpacks models. On the basis of these 
results, we propose a dispersed viscoelastic microsphere system and put forward five transport behaviors of low-elasticity microspheres in a porous medium. This research provides theoretical support for the further study of the dispersed viscoelastic microsphere system. The mechanism of improving oil recovery is proposed.

\section{Experimental section}

\subsection{Materials}

The materials applied were as follows: acrylamide (AM, purity above $98.5 \%$ ), acrylic acid (AA, purity above $99.7 \%$ ), ammonium persulfate (APS, purity above $98.0 \%$ ), dispersants (Span80 \& Tween60), ammonium chloride $\left(\mathrm{NH}_{4} \mathrm{Cl}\right.$, purity above $\left.99.5 \%\right)$, polyethylene glycol (PEG-200), sodium hydroxide $(\mathrm{NaOH}$, purity above $98 \%$ ), $N, N^{\prime}$-methylenebisacrylamide (MBA, purity above 99.0\%), anhydrous ethanol $\left(\mathrm{CH}_{3} \mathrm{CH}_{2} \mathrm{OH}\right.$, purity above $\left.99.5 \%\right)$, and acetone $\left(\mathrm{CH}_{3} \mathrm{COCH}_{3}\right.$, purity above $\left.99.5 \%\right)$. All the reagents were used without any purification. Hydrolyzed polyacrylamide (HPAM, relative molecular mass $27.4 \times 10^{6}$ ) was supplied by Dagang Oilfield (China), while aviation kerosene was purchased from the market. Deionized water was used for the preparation of all the aqueous solutions. The oil used in this study was from Dagang Oilfield (China) and was dehydrated crude oil. The composition of the simulated formation water used in the study is shown in Table 1.

\subsection{Preparation of low elastic microspheres}

The low-elastic microspheres were prepared by inverse suspension polymerization. Typically, $0.6 \mathrm{~g}$ of dispersants $[m($ Span 80$): m($ Tween 60$)=1: 1]$ and $60 \mathrm{~g}$ of aviation kerosene were put in a $250 \mathrm{~mL}$ four-necked flask equipped with a mechanical stirrer, a dropping funnel, a reflux condenser, and a nitrogen catheter. Meanwhile, the mechanical stirrer was started at a speed of $380 \mathrm{rpm}$ under nitrogen atmosphere to make the dispersants dissolve. Then, the mixed solutions (20 g) with AM, APS, MBA, $\mathrm{NH}_{4} \mathrm{Cl}$, PEG-200, and $\mathrm{NaOH}$ ( $6 \mathrm{~g}$ of AM, $0.0006 \mathrm{~g}$ of MBA, $0.2 \mathrm{~g}$ of $15 \mathrm{wt} \%$ APS, $0.34 \mathrm{~g}$ of $15 \mathrm{wt} \% \mathrm{NH}_{4} \mathrm{Cl}$, $0.005 \mathrm{~g}$ of PEG-200, and $0.254 \mathrm{~g}$ of $15 \mathrm{wt} \% \mathrm{NaOH}$ ) were added drop-wise into the oil phase at the speed of $2 \mathrm{~mL} \mathrm{~min}^{-1}$. The temperature of the thermostat water bath was raised up to $69^{\circ} \mathrm{C}$ after dropping off. After reaction for $6 \mathrm{~h}$ at this temperature, the resulting product was cooled and filtered and then dried at $80{ }^{\circ} \mathrm{C}$ for $48 \mathrm{~h}$. In this way, low-elastic microspheres were obtained.

\subsection{Microscopic characterization of the low-elasticity microspheres}

An XSJ-2 optical microscope (Chongqing Optical Instrument Co., Ltd., China) was used to observe the morphology of the low- elastic microspheres before and after swelling. FEI Quanta 200 FEG (FEI Company, Holland) was used to observe the internal structure of the low-elastic microspheres after swelling.

A Rize2006 laser particle size analyzer (Jinan Runzhi Technology Co., Ltd., China) was used to analyze the particle size before and after swelling. The initial particle size was measured in anhydrous ethanol solution, while the swollen particle size was measured in Dagang Oilfield simulated formation water at $50{ }^{\circ} \mathrm{C}$. In the particle size distribution curves, $D_{50}$ was used to express the average particle size of the microspheres before and after swelling. $D_{50}$ is the particle size from the cumulative distribution curve for a probability of $50 \%$.

\subsection{Swelling property and viscoelasticity of the low-elastic microspheres}

2.4.1 Swelling ratio measurement. In this paper, the swelling ratio (SR) was used to evaluate the swelling property of the low-elastic microspheres at different temperatures. The swelling ratio was calculated by eqn (1):

$$
\mathrm{SR}=\left(\frac{D_{t}}{d_{0}}\right)^{3}
$$

where SR is the swelling ratio of the low-elastic microspheres (in units of $\left.\mu \mathrm{m}^{3} \mu \mathrm{m}^{-3}\right), d_{0}$ is the initial average particle size of the low-elastic microspheres (in units of $\mu \mathrm{m}$ ), and $D_{t}$ is the average particle size of the low-elastic microspheres at swelling time $t$ (in units of $\mu \mathrm{m}$ ).

2.4.2 Viscoelasticity measurement. The temperature was controlled at a constant $50{ }^{\circ} \mathrm{C}$, and the viscoelasticity of the swollen low-elastic microspheres was measured using the Physica MCR301 Rheometer (Anton Paar, Austria). A flat-plate measuring system with a diameter of $43 \mathrm{~mm}$ was used. The gap was set at half of the average particle size of the swollen microspheres. Then, frequency sweep measurements were performed in the linear viscoelastic regime with the frequency from 0.01 to $10 \mathrm{~Hz}$ to determine the storage modulus $G^{\prime}$ and the loss modulus $G^{\prime \prime}$.

\subsection{Stability of the dispersed low-elastic microsphere system}

The stability of the dispersed low-elastic microsphere system was evaluated using a Turbiscan Lab Expert type stability analyzer manufactured by Formulation (France). Fig. 1 presents the measurement principle diagram from the Turbiscan Lab Expert type stability analyzer. This instrument was based on multiple light scattering. The transmittance $(T)$ and backscattering (BS) of a pulsed near-infrared light $(\lambda=880 \mathrm{~nm})$ were monitored by two synchronous optical detectors. The intensities of the reflected transmitted light and back-scattered light vary with the height of the sample. The scanning curve also

Table 1 Dagang Oilfield simulated formation water

\begin{tabular}{llllllll}
\hline Composition & $\mathrm{Na}^{+} / \mathrm{K}^{+}$ & $\mathrm{Ca}^{2+}$ & $\mathrm{Mg}^{2+}$ & $\mathrm{Cl}^{-}$ & $\mathrm{CO}_{3}{ }^{2-}$ & $\mathrm{HCO}_{3}^{-}$ & $\mathrm{SO}_{4}{ }^{2-}$ \\
\hline Concentration, $\mathrm{mg} \mathrm{L}^{-1}$ & 2043 & 39 & 36 & 1337 & 135 & 3126 & 10
\end{tabular}




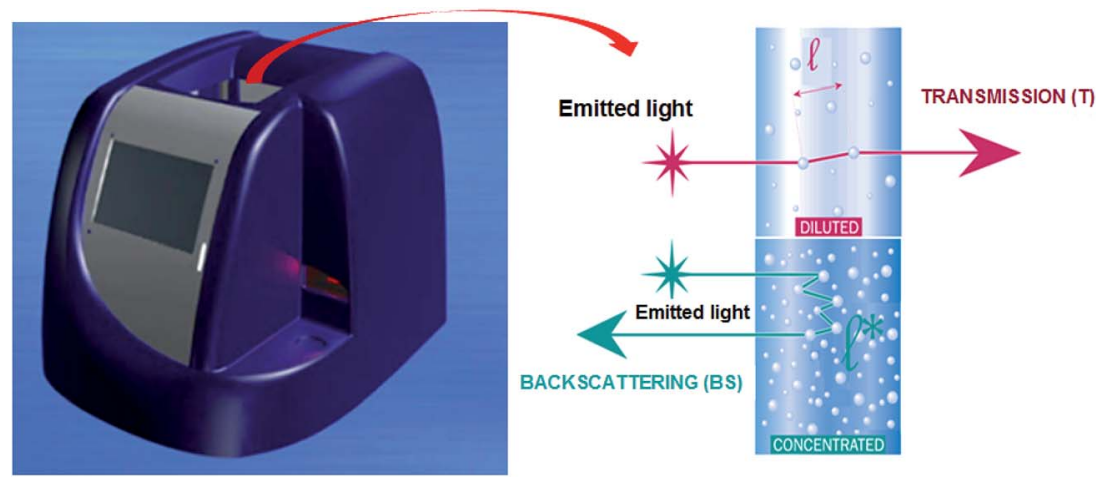

Fig. 1 Measurement principle diagram of the Turbiscan Lab Expert type stability analyzer.

reflected microscopic characteristics of the particle size growth or the migration of the colloidal or dispersed phase at a given time. The analysis of stability was carried out according to variations in the profiles of transmittance $(\mathrm{T})$ or backscattering (BS). The stability of the dispersed low-elastic microsphere system was evaluated by TSI (Turbiscan Stability Index) with the help of Turbiscan Easy Soft. TSI was calculated by eqn (2):

$$
\mathrm{TSI}=\sqrt{\frac{\sum_{i-1}^{n}\left(x_{i}-x_{\mathrm{bs}}\right)^{2}}{n-1}}
$$

where TSI represents the Turbiscan Stability Index, $n$ is the scan times, $x_{i}$ is the backscattering light intensity at the scanning time of $i$, and $x_{\mathrm{bs}}$ is the average backscattering light intensity.

TSI takes into account all the processes taking place in the sample (particle coalescence and settling process), so it is mainly used for the quantitative evaluation of dispersion stability. The larger the TSI value, the less stable the dispersed system. In this study, $20 \mathrm{~mL}$ of different dispersed low-elastic microsphere systems were placed into cylindrical glass tubes and submitted to analysis by the Turbiscan Lab Expert type stability analyzer. Two detectors were used to scan along the height of the different dispersed low-elastic microsphere systems sealed in the tubes at different temperatures for $2 \mathrm{~h}$.

\subsection{Rheological properties of the dispersed low-elastic microsphere systems}

The temperature was controlled at a constant $50{ }^{\circ} \mathrm{C}$. Rheological properties of different low-elastic microspheres systems were measured using a steady shear model and using the Physica MCR301 Rheometer (Anton Paar, Austria). A cylinder measuring system with an inner diameter of $26.666 \mathrm{~mm}$ was used. The steady shear measurement was conducted with a shear rate from 0.001 to $4000 \mathrm{~s}^{-1}$.

\subsection{Profile control and oil-displacement experiments}

2.7.1 Parallel-sandpacks test. Fig. 2 shows a schematic of the parallel-sandpacks flow test. The experimental setup consisted of a syringe pump, three cylinders, two sandpacks, and a pressure transducer connected to a desktop computer for continuous recording of the inlet pressure. The syringe pump (Model 260D, Teledyne ISCO, Lincoln, NE) was used to inject

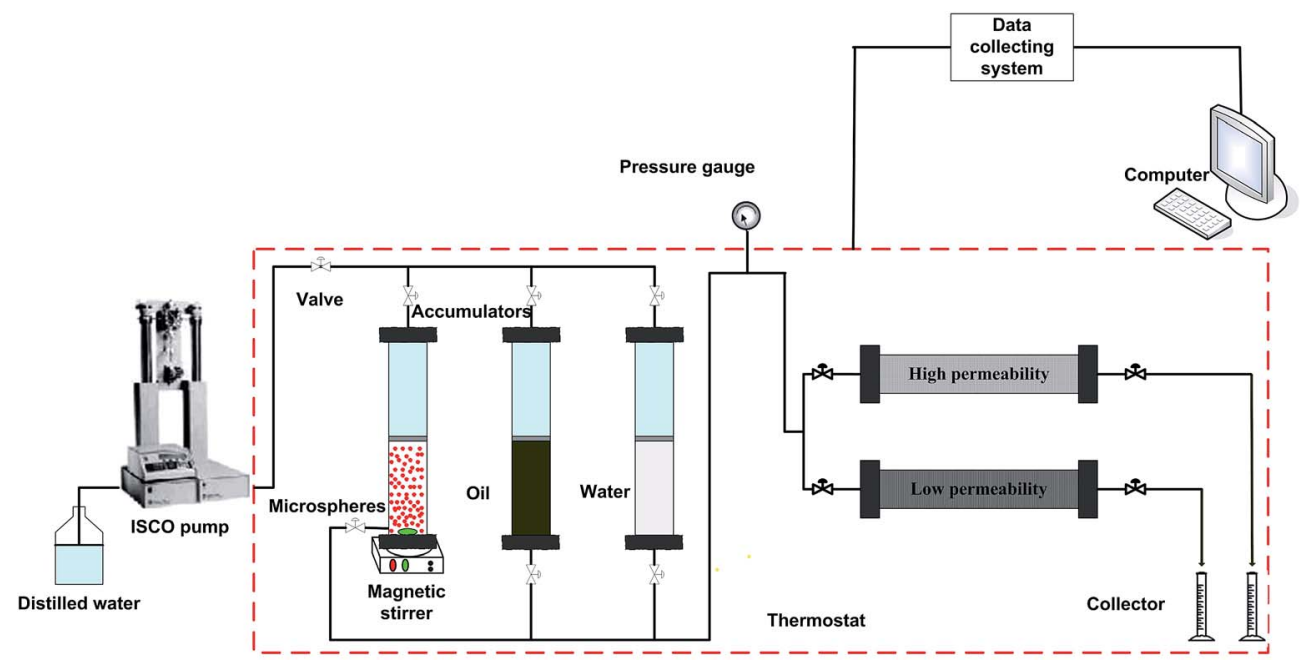

Fig. 2 Schematic of the parallel-sandpacks test for the dispersed low-elastic microsphere systems. 
fluids at a desired flow rate. All the parallel-sandpacks tests were conducted at $50{ }^{\circ} \mathrm{C}$.

Sandpacks were prepared at the ambient temperature of $25{ }^{\circ} \mathrm{C}$. Fresh sand was used for each test to ensure the same wettability. The porosity was measured using the weight method, while the permeability measurement was conducted using Darcy's law of single water flow. In the dispersed lowelastic microsphere flow tests, brine was first injected into the parallel sandpacks, followed by $0.4 \mathrm{PV}$ polymer flooding, at a concentration of $1000 \mathrm{mg} \mathrm{L} \mathrm{L}^{-1}$, using an ISCO pump, at a rate of $0.5 \mathrm{~mL} \mathrm{~min}^{-1}$. After that, water flooding was performed, followed by applying the dispersed low-elastic microsphere system, using an ISCO pump, at a rate of $0.5 \mathrm{~mL} \mathrm{~min}^{-1}$. After 0.4 PV dispersed low-elastic microspheres were injected, brine was injected in order to see the variation in the flow rates of the two sandpacks. The brine injection rate during these experiments was the same as that of the dispersed low-elastic microsphere system injection. The data for the pressures and effluent volumes of the two sandpacks were recorded.

2.7.2 Micromodel test. The micromodel was first cleaned using solvents and water. To ensure that the model was strongly water-wet, it was heated in an oven at $400{ }^{\circ} \mathrm{C}$, for $1 \mathrm{~h}$, to remove any organic material left from the previous test and solvent- cleaning process. To begin a test, the cleaned model was first vacuumed, and then saturated with brine. Oil was injected into the model to set the initial oil saturation. After the model was saturated with the oil, an initial water flooding was carried out using the synthetic formation water. At water flooding residual oil saturation, polymer and dispersed low-elastic microsphere system injection were carried out in sequence. A syringe pump was used to inject the fluids. The injection rate in all the stages

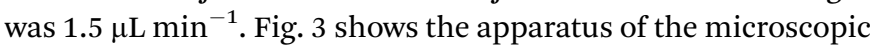
flooding experiment and the two-dimensional (2D) microscopic model with a pore throat diameter from $50 \mu \mathrm{m}$ to $200 \mu \mathrm{m}$.

\section{Results and discussion}

\subsection{Morphology and microstructure characterization of the low-elastic microspheres}

Fig. 4 gives the particle size analysis of the low-elastic microspheres before and after swelling at room temperature. It can be observed that the average particle sizes of the low-elastic microspheres before and after swelling are $100.5 \mu \mathrm{m}$ and $295.8 \mu \mathrm{m}$. As can be seen from Fig. 4, the low-elastic microspheres are spherical particles and can swell many times due to absorbing a lot of water. As the low-elastic microspheres

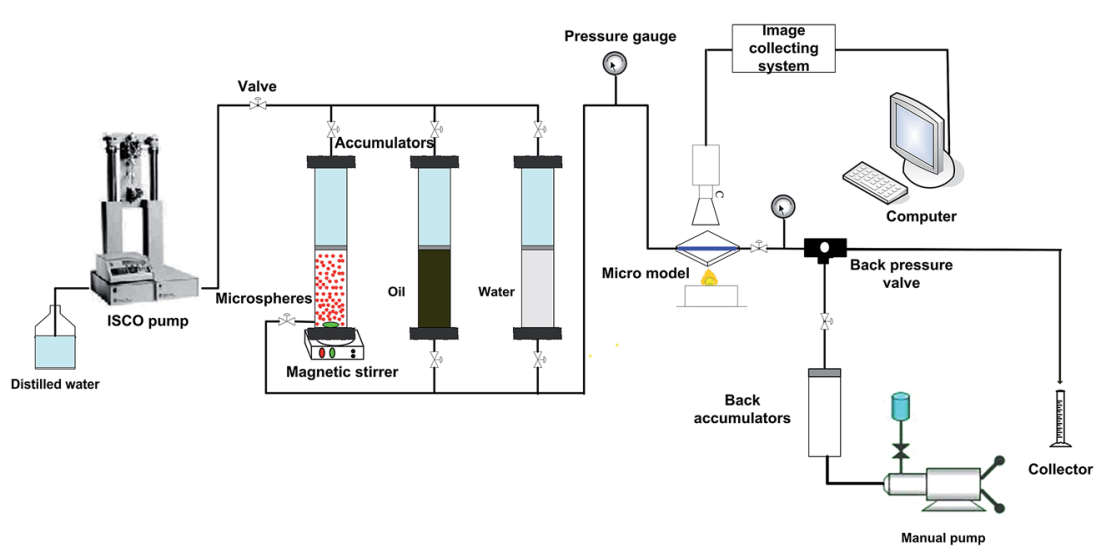

(a)

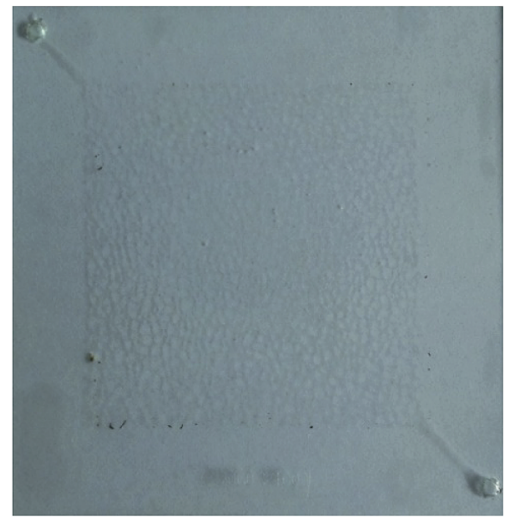

(b)

Fig. 3 (a) Apparatus of the microscopic flooding experiment and (b) two-dimensional microscopic model with a pore throat diameter from 50 $\mu \mathrm{m}$ to $200 \mu \mathrm{m}$ 


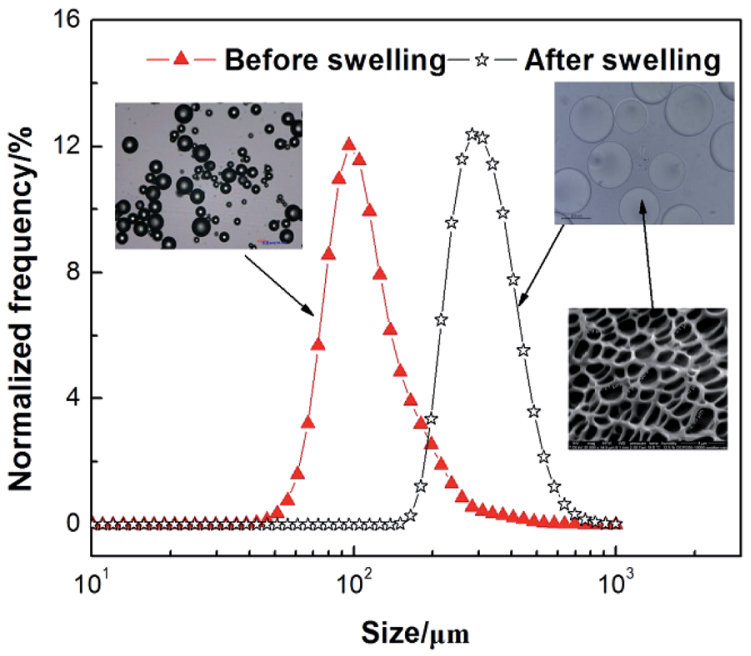

Fig. 4 Morphology and microstructure of the low-elastic microspheres.

contact with water, the water molecules can go into the internal network under the action of inside and outside osmotic pressure, stretching the molecular chain of the network structure and enlarging the volume absorbed by the low-elastic microspheres. The three-dimensional (3D) network structure with pore sizes ranging from $1.29 \mu \mathrm{m}$ to $2.95 \mu \mathrm{m}$ can be seen clearly from the ESEM image (Fig. 4). The investigated microstructure confirms the swelling mechanism of the low-elastic microspheres.

\subsection{Viscoelasticity and swelling property of the low-elastic microspheres}

3.2.1 Swelling property. The swelling capacity of the lowelastic microspheres was measured in the condition of simulated formation water and $50{ }^{\circ} \mathrm{C}$ at consecutive time intervals. Fig. 5 represents the dynamic swelling behavior of a low-elastic microsphere. Initially, the rate of swelling capacity sharply increases and then begins to level off. The final equilibrium swelling capacity of $21.71 \mathrm{~g} \mathrm{~g}^{-1}$ is achieved after $48 \mathrm{~h}$. A power law model is obvious from Fig. 5. The swelling kinetics can be expressed by the Voigt-based viscoelastic model in eqn (3):

$$
\mathrm{SR}_{t}=\mathrm{SR}_{\mathrm{e}}\left(1-\mathrm{e}^{-t / \tau}\right)
$$

where $\mathrm{SR}_{t}\left(\mathrm{~g} \mathrm{~g}^{-1}\right)$ is the degree of swelling at time $t, \mathrm{SR}_{\mathrm{e}}$ is the equilibrium swelling (power parameter, $\mathrm{g} \mathrm{g}^{-1}$ ), $t$ is the time (h) for swelling $\mathrm{SR}_{t}$, and $\tau(\mathrm{h})$ stands for the "rate parameter," denoting the time required to reach 0.63 of equilibrium water absorbency.

The data obtained from the equilibrium water absorbency and the swelling rate was fitted into eqn (3) to obtain the rate parameter and power parameter $\mathrm{SR}_{\mathrm{e}}$. The rate parameter and power parameter for the superabsorbent were found to be $3.24 \mathrm{~h}$ and $21.92 \mathrm{~g} \mathrm{~g}^{-1}$, respectively. Because the rate parameter $\tau$ is a measure of the resistance to water permeation, the relative value $(3.24 \mathrm{~h})$ reflects the water absorption rate.

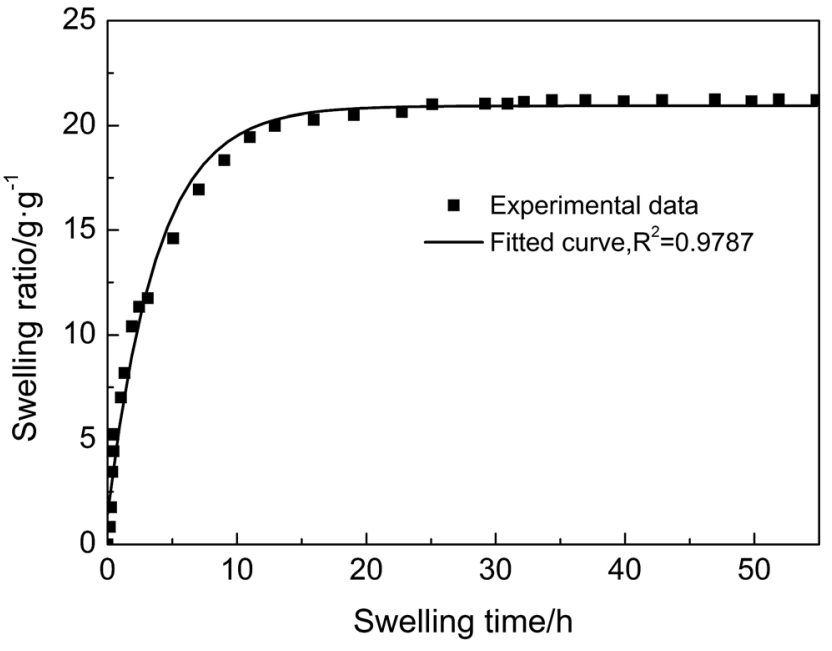

Fig. 5 Swelling property of the low-elastic microspheres.

3.2.2 Storage modulus $\left(G^{\prime}\right)$ and loss modulus $\left(G^{\prime \prime}\right)$. Fig. 6 shows the storage modulus $\left(G^{\prime}\right)$ and loss modulus $\left(G^{\prime \prime}\right)$ of prepared low-elastic microspheres. From the figure, we can see that $G^{\prime}$ and $G^{\prime \prime}$ of the low-elastic microspheres were 23.6 Pa and $2 \mathrm{~Pa}$. Through the figure, we can also see that $G^{\prime}$ is greater than $G^{\prime \prime}, G^{\prime}$ is much lower than the conventional microsphere $\left(G^{\prime}>\right.$ $100 \mathrm{~Pa})$, and the low-elastic microspheres showed the property of a soft solid. The main explanation for this is that the lowelastic microspheres can be deformed in the pore throat after swelling. In the application of the microspheres, the viscoelasticity of the microspheres should be adjusted according to the actual need; both to guarantee the shear resistance and ensure the strength of plugging.

\subsection{Stability of dispersed low-elastic microsphere systems}

3.3.1 Polymer concentration. The influence of polymer concentration on the stability of the dispersed low-elastic

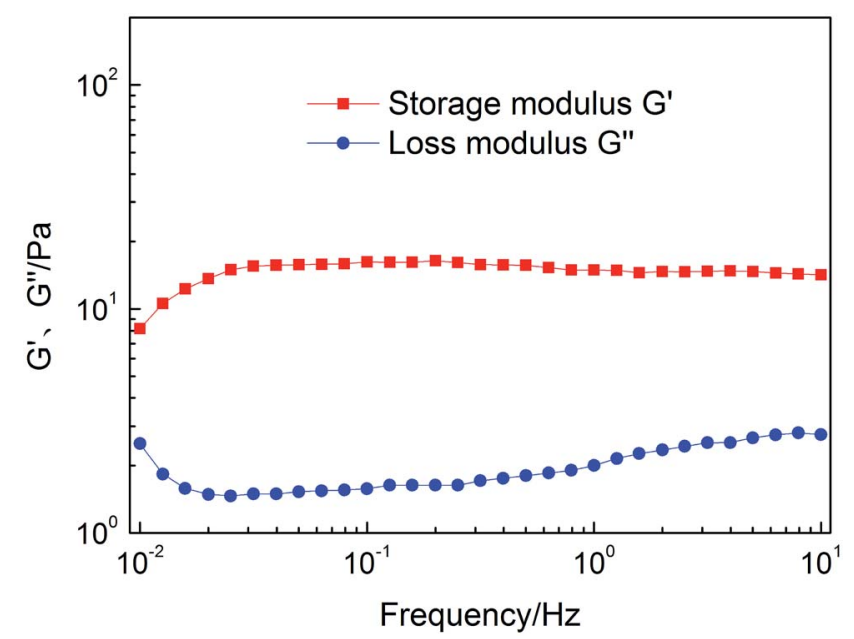

Fig. 6 Storage modulus $\left(G^{\prime}\right)$ and loss modulus $\left(G^{\prime \prime}\right)$ of the prepared low-elastic microspheres. 
microsphere system under the condition of $50{ }^{\circ} \mathrm{C}$ is shown in Fig. 7, whereby with the increase of polymer concentration, the stability of the oil-displacement system becomes better. That is because the polymer can increase the viscosity of the aqueous phase and increase the internal friction of the fluid, so that the TSI value gradually becomes smaller and the stability of the system becomes better. When the amount of polymer is $0.1 \%$, the TSI value is at a low level, and the back-scattered light and transmitted light change little with time, indicating that when the polymer concentration is $0.1 \%$, the stability of dispersed low-elastic microsphere system is good. Consequently, for the subsequent experimental procedures, the chosen polymer concentration was $0.1 \%$.

3.3.2 Formation temperature. The influence of formation temperature on the stability of the dispersed low-elastic microsphere system is shown in Fig. 8, whereby, with the increase of formation temperature, the TSI value of the dispersed low-elastic microsphere system increases, and the stability of the oil-displacement system becomes worse. This is primarily because, the higher the temperature of the system, the smaller the bulk viscosity of the dispersion medium, making the low-elastic microspheres easier to sink, resulting in deterioration of the stability of the whole system. During the construction process of an oil-displacement system, it is thus necessary to pay attention to the influence of formation temperature on the stability of the system. The stability of an oil-displacement system should be guaranteed so that the oildisplacement system can go into the deep reservoir and realize the purpose of deep profile control.

\subsection{Rheological properties of the dispersed low-elastic microsphere system}

Fig. 9 shows the apparent viscosity of the dispersed system with the increase of shearing rate at different polymer concentrations. As Fig. 9 shows, there is a critical shear rate $\left(\gamma_{c}\right)$ with the increase in shear rate. The apparent viscosity of the dispersed low-elastic microspheres system decreases with the increase in

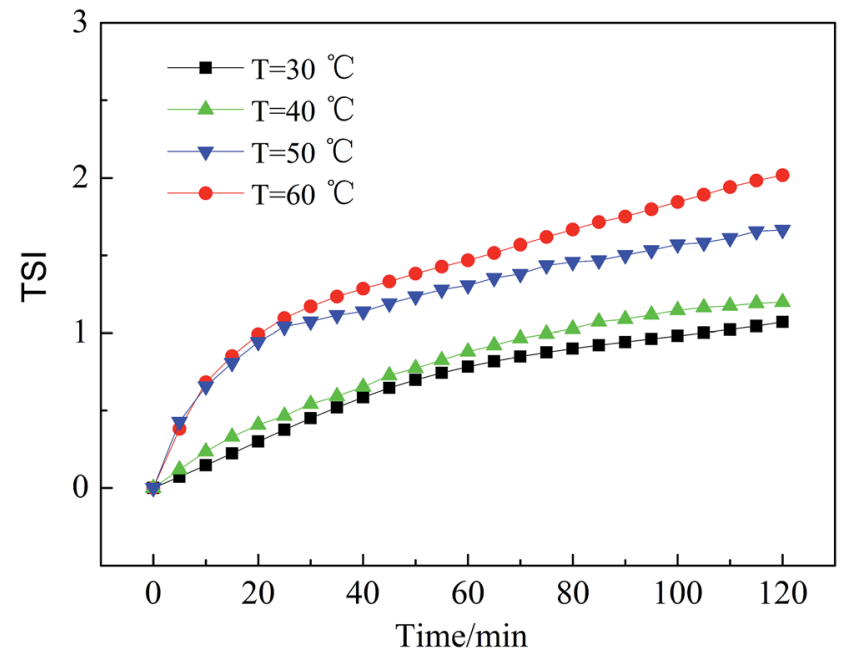

Fig. $8 \mathrm{TSI}$ of the dispersed low-elastic microspheres system at different formation temperatures.

shear rate in the case of $\gamma\left\langle\gamma_{c}\right.$, but the opposite is true when $\left.\gamma\right\rangle$ $\gamma_{\mathrm{c}}$. The former is classed as shear-thinning, and the latter shearthickening. At low shear rates, low-elastic microspheres in the dispersed system have a tendency to move away from each other due to repulsive inter-particle electrostatic or Brownian forces, which stabilizes the dispersed system and helps maintain a low viscosity. As the shear rate increases, the hydrodynamic forces increase, which makes the low-elastic microspheres move closer. When the hydrodynamic lubrication forces become greater than the repulsive forces, the low-elastic microspheres aggregate into clusters. These aggregates limit the flow, and this can explain the strong increase of viscosity observed at a critical shear rate.

The shear-thickening behavior is the result of the microstructure change and the interaction of the internal forces of the dispersed system. Fig. 10 shows a schematic diagram of the shear-thickening property. According to the multilayer structure theory of Hoffmann, ${ }^{39}$ the whole dispersed system shows an
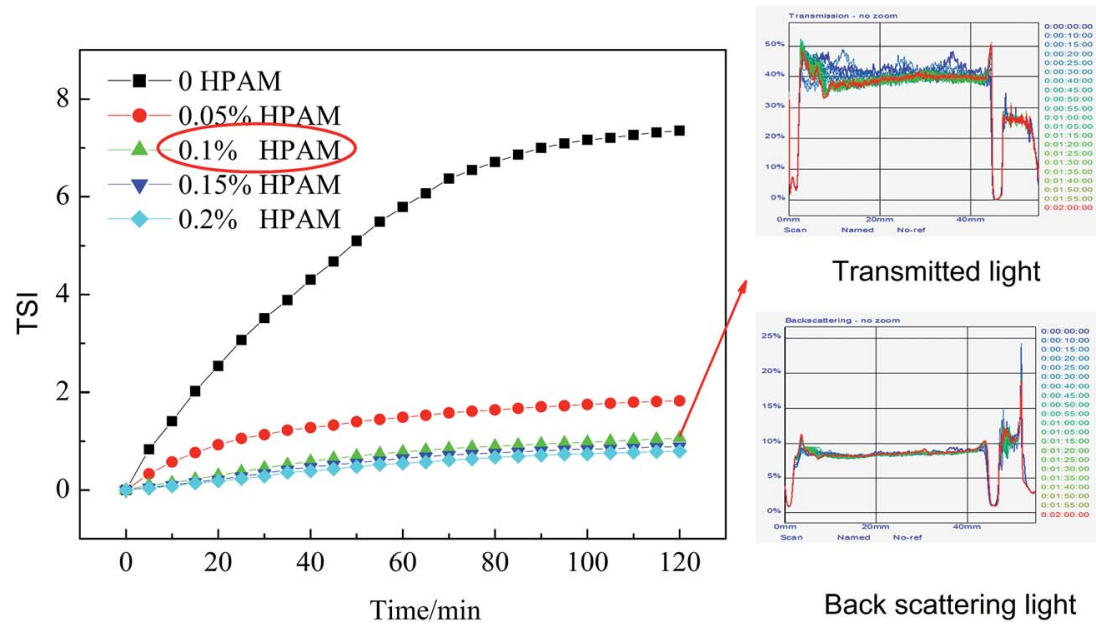

Fig. 7 TSI of the dispersed low-elastic microspheres system at different polymer concentrations. 


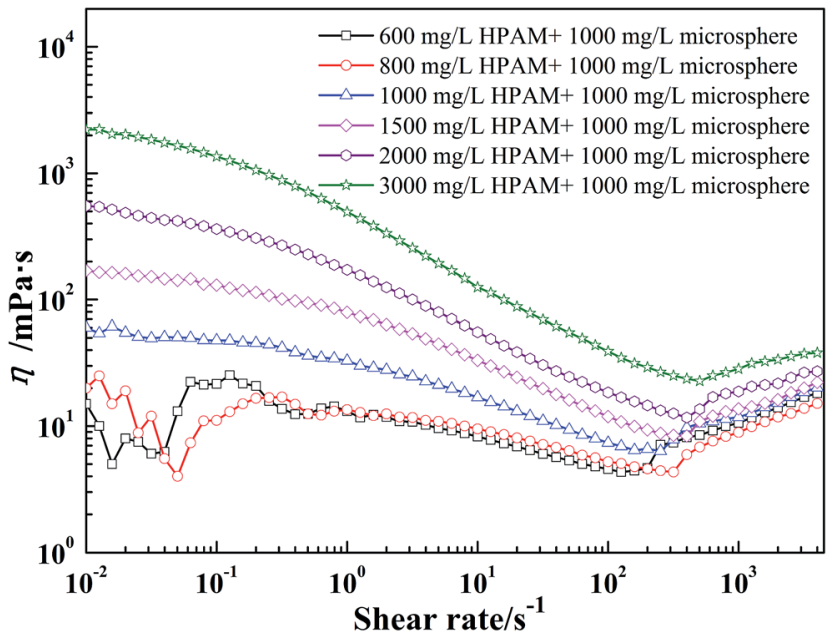

Fig. 9 The apparent viscosity of the dispersed system with the increase in shear rate at different polymer concentrations.

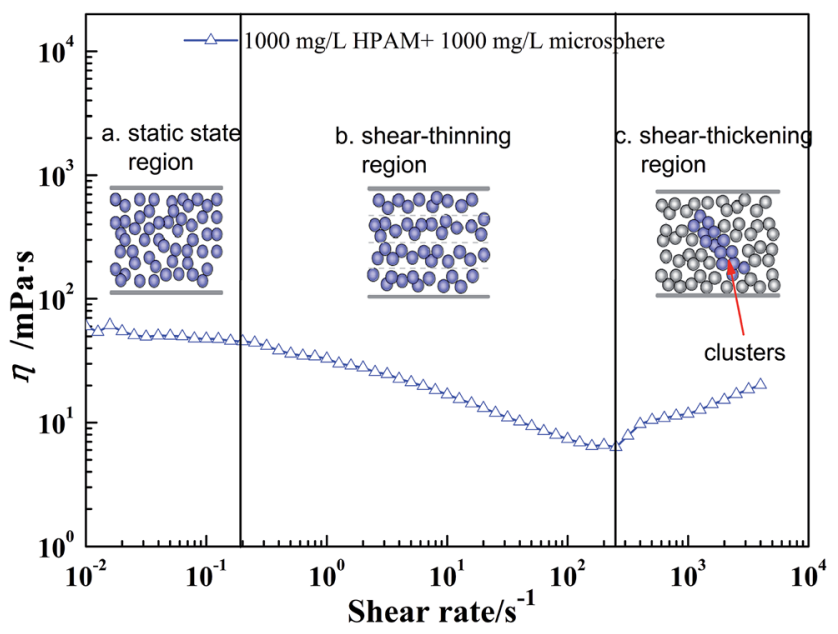

Fig. 10 Schematic of the shear-thickening property.

ordered layer structure at a low shear rate, where low-elastic microspheres flow in their respective layer separately. With the increase in shear rate, low-elastic microspheres only transform and orientate in their respective layers under shear, which leads to a shear-thinning behavior. The hydrodynamic force is the primary force in the dispersed system, which urges the isolated low-elastic microspheres to aggregate again and to form unsteady clusters separated by the dispersion medium. The clusters grow larger as the hydrodynamic force increases, as a result of which, the resistance effect on the system increases, thus showing an increase in the apparent viscosity. ${ }^{40-42}$

\subsection{Oil-displacement mechanism of the dispersed low- elastic microsphere system}

3.5.1 Micro-migration mechanism of the low-elastic microspheres. Microscopic displacement experiments observed that the low-elastic microspheres had five transport behaviors in porous media:
3.5.1.1 Deformable passing through. When the low-elastic microspheres are blocked in the pore throat, if the differential pressure of the driving fluid is high enough, it will squeeze the microspheres, and the swollen microspheres will dehydrate, shrink, and deform, such that they can then pass through the pore throat. When the deformed microspheres pass through the pore throat, they will absorb water again, and reform to their original sizes, as shown in Fig. 11(a).

3.5.1.2 Partition passing through. When the microspheres are plugged in the pores, if the differential pressure is high enough and the rate of compression is much larger than the rate of shrinkage, then the low-elastic microspheres will be cut off into smaller ones by the pore throat under the action of the pressure, as shown in Fig. 11(b).

3.5.1.3 Blockage. The low-elastic microspheres cannot move forward and so plug at the entrance of the pore throat when the diameter of the microspheres is larger than the pore throat size, because the differential pressure is not high enough to push the microspheres through the pore throat, as shown in Fig. 11(c).

3.5.1.4 Adhesion. When the low-elastic microspheres are too small, and the pressure is not sufficient to overcome the force of surface adsorption in porous media particles, the low-elastic microspheres will adhere and be retained on the surface of the porous medium, with these low-elastic microspheres mainly located between the main channels, as shown in Fig. 11(d).

3.5.1.5 Direct passing through. Here, the low-elastic microspheres are smaller than the pore throat diameter, and the pressure is greater than the force of the surface adsorption in porous media particles, as shown in Fig. 11(e).

3.5.2 Oil-displacement mechanism of the dispersed lowelastic microsphere system

3.5.2.1 Oil-displacement effect and oil-displacement mechanism. As shown in Fig. 12, due to a high water-oil mobility ratio, the swept volume of water flooding is small and water preferentially accesses large pores and a fingering phenomenon occurs, whereas small holes are not swept and a lot of remaining oil is hence distributed there. The remaining oil is mainly distributed in small pores in a low permeability zone, with less large pores, as shown in Fig. 12(a). Polymer flooding can reduce the mobility ratio effectively due to the high viscosity of the polymer, so that it increasing the swept volume and decreases the remaining oil by spreading it into the small pores, as shown in Fig. 12(b). Compared with polymer flooding and water flooding, combination flooding (dispersed low-elastic microsphere system) performs better in oil displacement, because the microspheres can effectively block the large pores and make the liquid flow change direction, which means it can be spread to the low permeability zone better, as shown in Fig. 12(c).

According to the definition of oil-displacement efficiency, the microscopic oil-displacement efficiency $E_{\mathrm{R}}$ can be expressed as:

$$
E_{\mathrm{R}}=\frac{\mathrm{As}_{\mathrm{wor}}-\mathrm{As}}{\mathrm{As} \mathrm{s}_{\mathrm{wor}}}
$$

where $E_{\mathrm{R}}$ is the microscopic oil-displacement efficiency (\%) and $\mathrm{As}_{\mathrm{wor}}$ and $\mathrm{As}_{\text {or }}$ are the original oil-bearing area and remaining oil area, separately. 


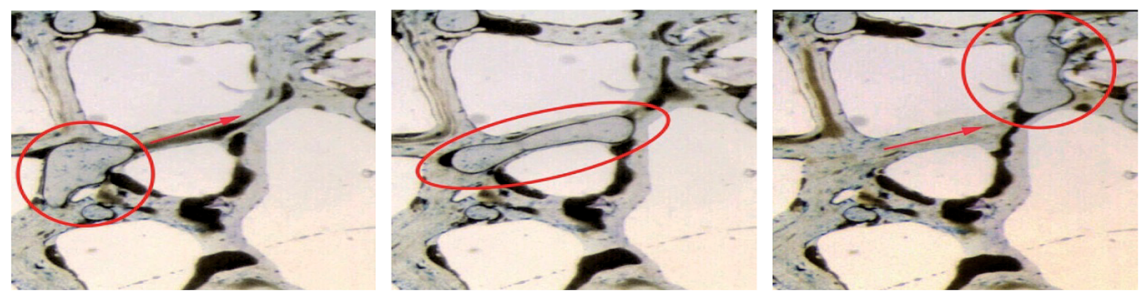

(a) Deformable passing through
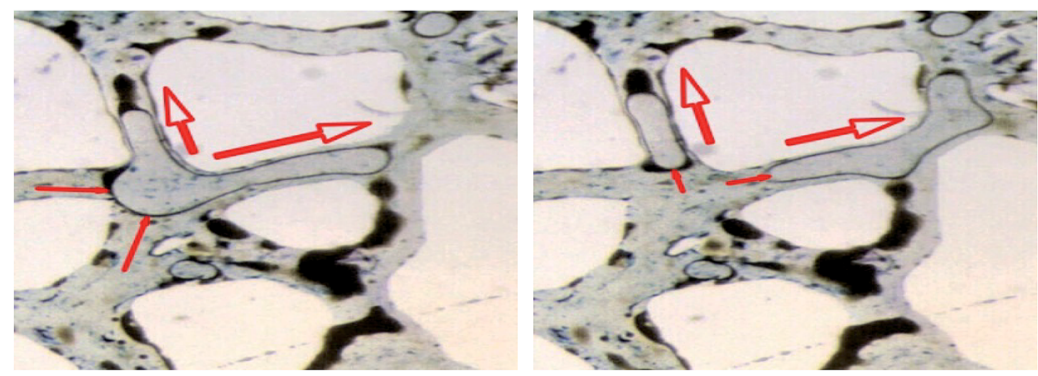

(b) Partition passing through

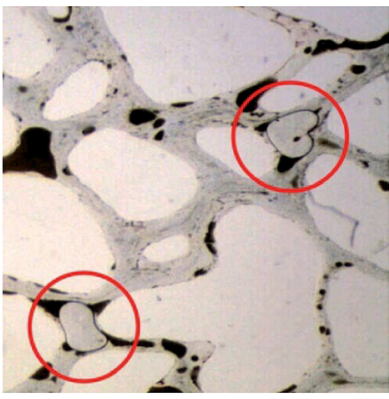

(c) Blockage

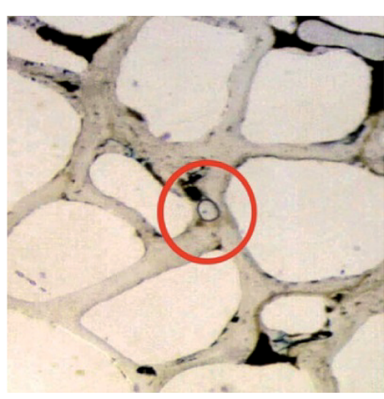

(d) Adhesion

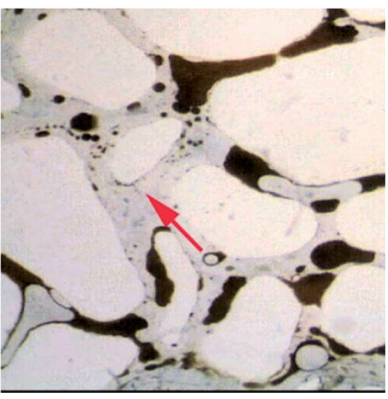

(e) Direct passing through

Fig. 11 Transport behaviors of low-elastic microspheres in a porous medium.

The auxiliary line and area method were used to quantitatively characterize the oil-displacement efficiency after different flooding simulations. According eqn (4), we calculated the microscopic oil-displacement efficiency after water flooding and polymer flooding as $51.82 \%$ and $69.37 \%$, while the microscopic oil-displacement efficiency after combination flooding was up to $91.24 \%$. By comparing the distribution of the remaining oil and the microscopic oil-displacement efficiency after different flooding simulation, we can see the effect of oil displacement, whereby: combination flooding > polymer flooding $>$ water flooding.

Analyzing the microscopic oil-displacement data, which was obtained in microscopic visualization oil-displacement experiments, allowed us to summarize the flooding mechanism of the dispersed low-elastic microsphere system by the following aspects: (1) selective blocking mechanism; (2) deep flow steering mechanism; (3) microsphere elastic deformation flooding mechanism; (4) mechanism of polymer flooding.

(1) Selective blocking mechanism. In the microscopic model, a water channel can form after water flooding. This has a low flow resistance, so that when the dispersed low-elastic microsphere system is injected, it is easier to migrate into these large pores. Low-elastic microspheres fill the large pores and rarely enter the small pores, increasing the flow resistance of the large pores and blocking the high permeability zone effectively, thus reducing the permeability.

(2) Deep flow steering mechanism. After entering the large pores, the dispersed low-elastic microsphere system can migrate forward given the larger injection pressure differential. It can pass through directly when the size of the low-elastic microsphere is smaller than the large pore diameter. When the particle size of the low-elastic microsphere is greater than the pore diameter, it can be deformed or cut off at a sufficient pressure, and then it will continue to migrate to the deep zone. After the deep formation pressure differential decreases, the smaller microspheres are adsorbed on the pore. It is possible to generate additional flow resistance of the fluid by changing the flow line, so that it spreads the formation better, as shown in Fig. 11(c) and (d). As for the larger size particles, the reduced pressure difference with the deep reservoir is not enough to push through the low-elastic microspheres through the smaller pores, which will block the large pores, thereby reducing the 


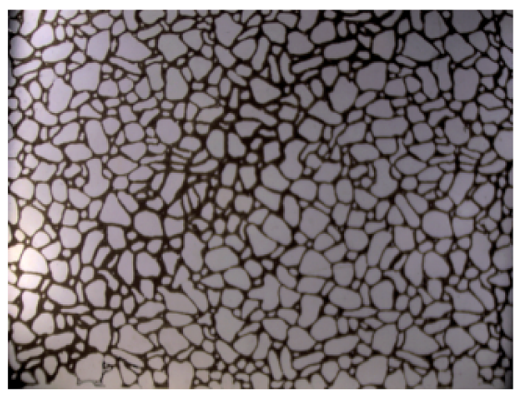

(a) Oil saturated

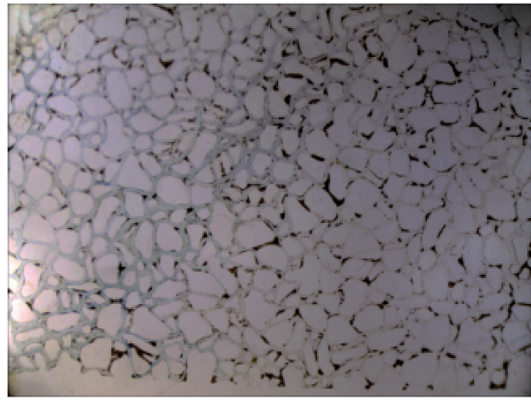

(c) After polymer flooding

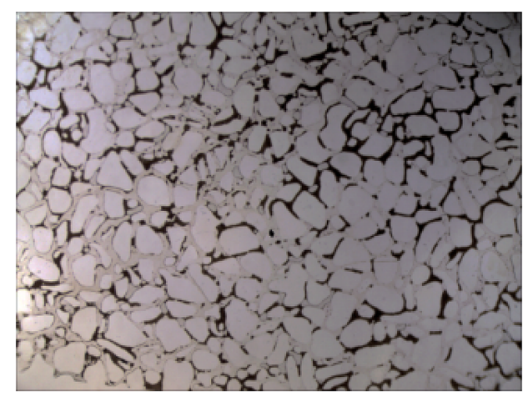

(b) After water flooding

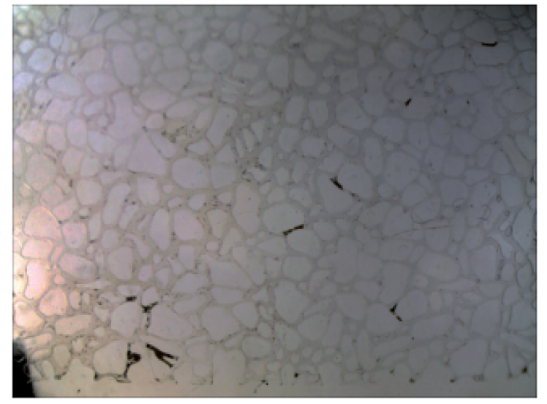

(d) After combination flooding

Fig. 12 Residual oil distribution after different displacements.

permeability, and then the liquid flow will turn into low permeability formations, increasing the spread area and enhancing the oil recovery, as shown in Fig. 11(b).

(3) Deformation displacement mechanism. When the particle size of the low-elastic microspheres was larger than the pore diameter, the low-elastic microspheres deformed into the pores elastically under the effect of fluid force and the microspheres were stretched. After passing through the pores, the action of squeezing and stretching from the pore disappears and the microspheres expand to their normal size in the process of shape restoration, and then migrate to the remaining oil zone that could not be swept before to push them out, as shown in Fig. 11(c).

(4) Mechanism of polymer flooding. The dispersed low-elastic microsphere system with good shear-thickening ability can effectively decrease the water-oil mobility ratio in the progress of flooding and enhance the sweep efficiency. Due to the solvation and hydrolysis of the polymer in the combination system, the molecules entangled in the water can form a random coil. As a viscoelastic solution, the polymer solution can be stretched due to the structure of the pores when passing through the pore throat, and then re-curled when the stretching effect disappears. During this process, the polymer molecules can strip the residual oil in the pore throat.

From the research into the micro-migration mechanism and oil-displacement mechanism of the dispersed polymer microsphere system, we know that the micro-migration mechanism of polymer microspheres with different storage moduli is the integrative reflections of different transport behaviors. However, the transport behaviors of high elastic microspheres are mainly broken up by passing through blockages and by direct passing through. Because of the fragility of the swollen high elastic microspheres, deformable passing through and partition passing through are hard to find in the transport behaviors of high elastic microspheres. Also, the deformation displacement mechanism is not obvious during the oil displacement of high elastic microspheres. Thus, the dispersed low-elastic microsphere system shows good application prospects in the profile control and oil displacement of heterogeneous reservoirs.

3.5.2.2 EOR capacity evaluation. The oil recovery and injection pressure of two parallel-sandpacks tests with different permeability contrasts are plotted as a function of injection volume in Fig. 13. Both of the diagrams in Fig. 13 have three curves, representing the oil recovery of high permeability sandpack (red curve), the oil recovery of low permeability sandpack (blue curve), and the total oil recovery (green curve), respectively. Another curve is the injection pressure curve (black line). The oil recovery covered all five displacement stages: initial water flooding, $0.4 \mathrm{PV}$ polymer flooding, subsequent water flooding, 0.4 PV combination flooding, and subsequent water flooding.

The results of the two tests showed that the oil recovery of high permeability sandpacks was higher than those of low permeability sandpacks. The results also showed that this difference increases with the increase in permeability contrast of the two sandpacks. When the permeability contrast was 3 in Fig. 13(a), water flooding recovered $40.70 \%$ of the initial oil in place (IOIP) of the high permeability sandpack and 13.26\% IOIP of the low permeability sandpack. When the permeability contrast of the two sandpacks was increased to 20.4, the oil 


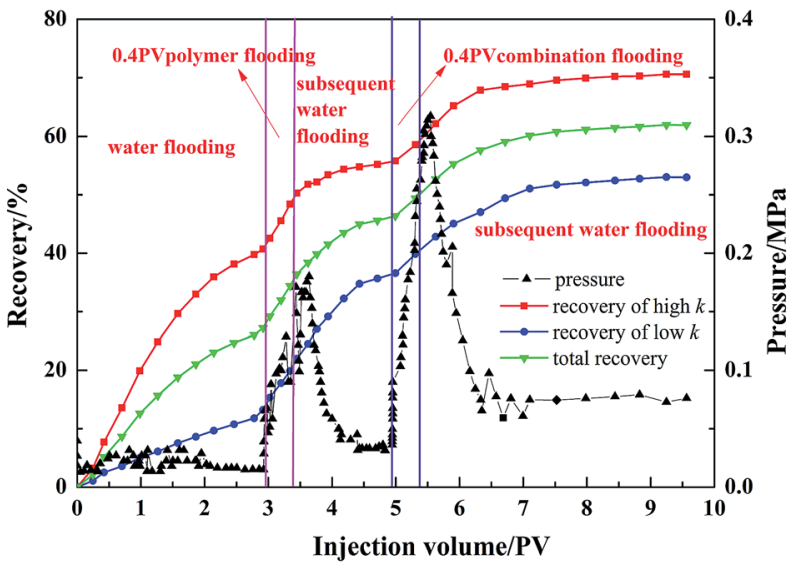

(a)

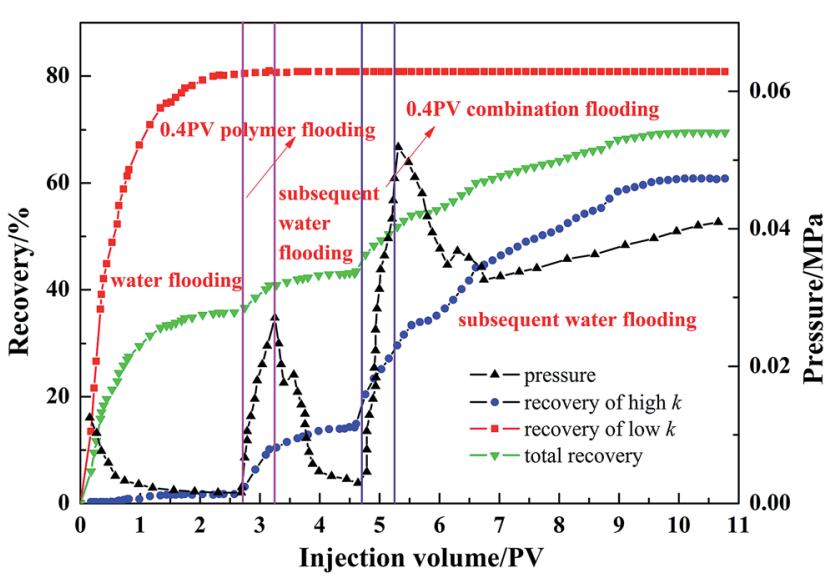

(b)

Fig. 13 Dynamic displacement curves of the dispersed low-elastic microsphere system in the parallel-sandpacks model with different permeability contrasts ((a) permeability contrast $=3$; (b) permeability contrast $=20.4$ ).

recovery of the high permeability sandpack was about $80.83 \%$, compared with $1.75 \%$ for the low permeability sandpack. This means that a high permeability contrast will result in high remaining oil saturation in the low permeability sandpack. The trends of the recovery curves at the end of initial water flooding showed that oil recovery in the low permeability sandpack would not catch up with the oil recovery of the high permeability pack by simply applying water flooding. Although polymer flooding was conducted, the poor oil-displacement effect was not improved so much.

The injection purpose of combination flooding was to improve the sweep efficiency of parallel sandpacks. The oil recovery curves of the two diagrams in Fig. 13 show that both recovery curves increased during the combination flooding, with the curve of the low permeability sandpack increasing much faster than that of the high permeability sandpack, indicating that the injected dispersed low-elastic microsphere system could divert the major fluid flow from the high permeability sandpack to the low permeability sandpack.

Because the majority of the injected low-elastic microspheres stayed in the high permeability sandpack, the profile control effect could still be reflected during the subsequent water flooding stage. This can be observed in the change in the oil recovery curves of the subsequent water flooding. For Fig. 13(a) and (b), with a $0.4 \mathrm{PV}$ dispersed low-elastic microsphere system injection and subsequent water flooding, the cumulative oil recoveries in the low permeability sandpack were $52.99 \%$ and $60.85 \%$ IOIP, respectively, which was even slightly higher than the cumulative oil recoveries in the high permeability packs: $70.58 \%$ and $80.83 \%$ IOIP for Fig. 13(a) and (b). It should be noted that the final oil recovery in the low permeability sandpack was very close to, but still a little bit lower than, that of the high permeability sandpack. The injection pressure curve showed that the injected low-elastic microsphere system could be forced into the high permeability sandpack, resulting in a relatively high flow resistance to the injection fluid in it.

The results presented in the last two columns of Table 2 show that the incremental recoveries by the dispersed lowelastic microsphere system from the low permeability packs are larger than those from the high permeability packs, especially the permeability contrast of 20.4. The permeability contrasts are 3 and 20.4, respectively. The total incremental recoveries compared with polymer recovery are $16.35 \%$ and $26.04 \%$, respectively. These results show that injection of the dispersed low-elastic microsphere system can effectively improve the sweep efficiency and displace more remaining oil in the low permeability zones. For a more heterogeneous model, the effect of the low-elastic microspheres on the sweep efficiency becomes more noticeable. This stems from the fact that the low-elastic microspheres not only increase the viscosity of the water phase, but also divert the flow to the upswept low permeability zones.

Table 2 Statistical table of the recovery in different stages

\begin{tabular}{lllllll}
\hline $\begin{array}{l}\text { Permeability } \\
\text { contrast }\end{array}$ & Model $/ \times 10^{-3} \mu \mathrm{m}^{2}$ & $\begin{array}{l}E_{\mathrm{R}} \text { after } \\
\text { water flooding/\% }\end{array}$ & $\begin{array}{l}E_{\mathrm{R}} \text { after polymer } \\
\text { flooding/\% }\end{array}$ & $\begin{array}{l}E_{\mathrm{R}} \text { after combination } \\
\text { flooding/\% }\end{array}$ & $\begin{array}{l}\Delta E_{\mathrm{R}} \text { compared with } \\
\text { water flooding/\% }\end{array}$ & $\begin{array}{l}\Delta E_{\mathrm{R}} \text { compared with } \\
\text { polymer flooding/\% }\end{array}$ \\
\hline 3 & Low $k(383.7)$ & 13.26 & 35.65 & 52.99 & 39.73 & 17.34 \\
& High $k(1138.1)$ & 40.70 & 55.20 & 70.58 & 29.88 & 15.38 \\
\multirow{5}{*}{20.4} & Total & 27.26 & 45.60 & 61.95 & 59.69 & 16.35 \\
& Low $k(1100)$ & 1.75 & 14.93 & 60.85 & 0 & 45.92 \\
& High $k(22400)$ & 80.83 & 80.83 & 80.83 & 33.51 & 26.04
\end{tabular}




\section{Conclusions}

In this study, a novel dispersed low-elastic microspheres system for enhanced oil recovery in heterogeneous reservoirs was proposed. Laboratory experiments were conducted to understand the stability, rheological property, and oil-displacement mechanism of dispersed low-elastic microspheres. The major conclusions that can be drawn from this study are as follows:

(1) The dispersed low-elastic system, which is formed by lowelastic microspheres and polymers, has good stability and exhibits a characteristic "shear-thickening" behavior. The stability of the dispersed system is influenced by many factors. The higher the concentration of the polymer is, the more stable the system is, with the optimal system being composed of $0.1 \%$ low-elastic microspheres and $0.1 \%$ polymer HPAM. In the weak alkaline environment, the higher the temperature is, the more stable the system is. The "shear-thickening" behavior of dispersed low-elastic microspheres system was the result of the microstructure change and the internal forces of the dispersed system.

(2) The low-elastic microspheres have good deformability in porous media. Microscopic displacement experiments showed that the transport behaviors of low-elastic microspheres in porous media are deformable passing through, partition passing through, blockage, adhesion and direct passing through, respectively. The EOR results in the micromodel test and in the parallel-sandpacks test show that the injection of the dispersed low-elastic microspheres system can effectively improve the sweep efficiency and recover more residual oil in the low permeability zones. For a larger permeability contrast model, the effect of the dispersed low-elastic microspheres system on the sweep efficiency becomes more noticeable. Thus, the oil-displacement mechanism of the dispersed low-elastic microsphere system can be summarized as follows: selective plugging mechanism, deep flow steering mechanism, deformation displacement mechanism, mechanism of polymer flooding.

\section{Conflict of interest}

The authors declare no competing financial interest.

\section{Acknowledgements}

This research was financially supported by the National Natural Science Foundation of China (21273286, 51574267), the Fundamental Research Funds for the Central Universities (16CX06032A, 15CX08003A), Science Foundation of China University of Petroleum, Beijing (No. 2462015YJRC033) and Qingdao Postdoctoral Application Research Project (No. 2016222).

\section{References}

1 F. L. Zhao, Enhanced Oil Recovery Principles, China University of Petroleum Press, Dongying, PRC, 2000.

2 H. Q. Liu and H. L. Zhang, Pet. Sci., 2006, 3, 51.
3 R. B. Needham, C. B. Threlkeld and J. W. Gall, SPE4747 presented at SPE Improved Oil Recovery Symposium, 1974.

4 A. M. Araghi, J. Pet. Sci. Eng., 2000, 26, 1.

5 D. Perez, F. E. Fragachan, A. Ramirez and J. P. Ferraud, SPE37622 presented at Drilling Conference, 2001.

6 R. S. Seright, R. H. Lane and R. D. Sydansk, SPE70067 presented at Oil and Gas Recovery Conference, 2003.

7 G. Zhao, C. L. Dai, Q. You, M. W. Zhao and J. H. Zhao, J. SolGel Sci. Technol., 2013, 65, 392.

8 G. Zhao, C. L. Dai, M. W. Zhao and Q. You, J. Appl. Polym. Sci., 2014, 131, 1.

9 H. Zhang, S. C. Rajesh, B. J. Bai and J. L. Wang, Ind. Eng. Chem. Res., 2010, 49, 12284.

10 B. J. Bai, M. Z. Wei and Y. Z. Liu, SPE 164511 presented at SPE Production and Operations Symposium, 2013.

11 O. E. Mahmoud and B. J. Bai, SPE 164879 presented EAGE Annual Conference \& Exhibition incorporating SPE Europec, 2013.

12 G. Chauveteau, A. Omari, R. Tabary, M. Renard, J. Veerapen and J. Rose, SPE 64988 presented at SPE International Symposium on Oilfield Chemistry, 2001.

13 A. Zaitoun, R. Tabary, D. Rousseau, T. R. Pichery and S. Nouyoux, SPE 106042 presented at International Symposium on Oilfield Chemistry, 2007.

14 S. K. Choi, Y. M. Ermel, S. L. Bryant, C. Huh and M. M. Sharma, SPE 99656 presented at SPE/DOE Symposium on Improved Oil Recovery, 2006.

15 I. P. Benson, L. X. Nghiem, S. L. Bryant and C. Huh, SPE 109665 presented at SPE Annual Technical Conference and Exhibition, 2007.

$16 \mathrm{~J}$. Pritchett, H. Frampton and J. Brinkman, SPE 84897 presented at SPE International Improved Oil Recovery Conference in Asia Pacific, 2003.

17 H. Frampton, J. C. Morgan, S. K. Cheung, L. Muson, K. T. Chang and D. Williams, SPE 89391 presented at SPE/ DOE Symposium on Improved Oil Recovery, 2004.

18 B. Bai, F. Huang, Y. Liu, R. S. Seright and Y. Wang, SPE 113997 presented at SPE Symposium on Improved Oil Recovery, 2008.

19 R. Larkin and P. Creel, SPE 113305 presented at SPE Symposium on Improved Oil Recovery, 2008.

$20 \mathrm{H}$. Zhang and B. J. Bai, SPE J., 2011, 16, 388.

21 A. Al-Ibadi and F. Civan, SPE 153557 presented in SPE Latin America and Caribbean Petroleum Engineering Conference, 2012.

22 X. F. Jia, G. L. Lei and X. Y. Jia, Spec. Oil Gas Reservoirs, 2009, 16, 6.

23 C. J. Yao, G. L. Lei, L. Li and X. M. Gao, J. Appl. Polym. Sci., 2013, 127, 3910.

24 Z. Hua, M. Lin, Z. Dong, M. Li, G. Zhang and J. Yang, J. Colloid Interface Sci., 2014, 424, 67.

25 P. Li, J. P. Zhang and A. Q. Wang, Macromol. Mater. Eng., 2007, 292, 962.

26 T. Wan, R. Q. Huang, Q. H. Zhao, L. Xiong, L. Luo, X. M. Tan and G. J. Cai, J. Appl. Polym. Sci., 2013, 87, 698.

27 C. J. Yao, G. L. Lei, L. Li and X. M. Gao, Energy Fuels, 2012, 26, 5092. 
28 G. L. Lei and J. P. Zheng, J. China Univ. Pet., 2007, 31, 87.

29 C. Liu, X. Liao, Y. Zhang, C. Mu, T. Li, R. Qin, R. Fu, X. Bie and J. Zheng, SPE 158293 presented at SPE Annual Technical Conference and Exhibition, 2012.

30 Z. Hua, M. Lin, J. Guo, F. Xu, Z. Li and M. Li, J. Pet. Sci. Eng., 2013, 105, 70.

31 Y. C. Wu, B. Gao, X. Hou and K. D. Yao, Polym. Mater. Sci. Eng., 2008, 24, 13.

32 N. Azema, Powder Technol., 2006, 165, 133.

33 C. Lemarchand, P. Couvreur, C. Vauthier, D. Costantini and R. Gref, Int. J. Pharm., 2003, 254, 77.

34 R. Vie, N. Azema, J. C. Quantin, E. Touraud and M. Fouletier, Colloids Surf., A, 2007, 298, 192.
35 Y. F. Ji, W. L. Kang, L. W. Meng, L. L. Hu and H. B. Yang, Colloids Surf., A, 2014, 453, 117.

36 M. Balastre, J. F. Argillier, C. Allain and A. Foissy, Colloids Surf., A, 2002, 211, 145.

37 H. Yang, W. Kang, S. Liu, B. Bai, J. Zhao and B. Zhang, J. Dispersion Sci. Technol., 2015, 36, 1673.

38 H. Yang, W. Kang, Y. Yu, Y. Lu, Z. Li, M. Wang and T. Liu, J. Appl. Polym. Sci., 2015, 132, 42278.

39 R. L. Hoffmann, J. Rheol., 1998, 42, 111.

40 B. J. Maranzano and N. J. Wagner, J. Chem. Phys., 2002, 117, 10291.

41 H. Watanable, M. L. Yao, K. Osaki, T. Shikata, H. Niwa and Y. Morishima, Rheol. Acta, 1998, 37, 1.

42 B. J. Maranzano and N. J. Wagner, J. Rheol., 2001, 45, 1205. 